\title{
Optimization of processing parameters for vacuum fried oyster mushroom (Pleurotus ostreatus (Jacquin) P. kummer)
}

\author{
${ }^{1,2}$ Hilapad, M.R., ${ }^{3}$ Esguerra, E.B. and ${ }^{2 *}$ Castillo - Israel, K.A.T. \\ ${ }^{1}$ Capiz State University-Mambusao Satellite College, Mambusao, Capiz \\ ${ }^{2}$ Institute of Food Science and Technology, College of Agriculture and Food Science, University of the \\ Philippines, Los Baños, 4031, College, Laguna \\ ${ }^{3}$ Postharvest Horticulture Training and Research Center, College of Agriculture and Food Science, \\ University of the Philippines, Los Baños, 4031, College, Laguna
}

\author{
Article history: \\ Received: 13 February 2020 \\ Received in revised form: 6 \\ April 2020 \\ Accepted: 8 April 2020 \\ Available Online: 28 April \\ 2020
}

\section{Keywords:}

Central composite design, Oyster mushroom,

Response surface

methodology,

Total phenolics content,

Vacuum fried

\section{DOI:}

https://doi.org/10.26656/fr.2017.4(4).065

\begin{abstract}
This study is aimed to optimize the processing parameters for the production of a vacuum fried product from oyster mushroom (Pleurotus ostreatus (Jacquin) P. kummer). A $3^{3}$ factorial experiment was employed following the Central Composite Design (CCD) with fifteen experimental treatments. The treatments were subjected to proximate analysis, total phenolics content, DPPH assay and sensory evaluation. Contour plots of the results of various analyses were superimposed to identify the optimum process and formulation. Variables considered for optimization were maturity, salt concentration and frying time. Response surface regression analysis revealed that maturity significantly affected the crude protein, crude fiber, total phenolics content (TPC) and radical scavenging activity (RSA) while salt concentration significantly affected the ash, crude fat, crude fiber, TPC, aroma and crispiness of the vacuum fried product. On the other hand, frying time significantly affected the ash content. Interaction between variables also significantly affected ash, crude fat, crude fiber, TPC, RSA, crispiness and taste. Parameters considered in the identification of the optimum region were ash content, crude protein, crude fiber, radical scavenging activity and sensory attributes. Minimum values were set for each parameter as follows: $\geq 3 \%$ for ash, $\geq 11 \%$ for crude protein, $\geq 10 \%$ for crude fiber, and $\geq$ 6.75 for sensory attributes. Superimposed contour plots showed that the optimum region based on set of criteria is at treatment combination of 3 days fruiting body maturity, $3 \%$ salt concentration and 35 mins vacuum frying time. Verification test reveals that the optimum vacuum fried product was highly acceptable than the product found outside the optimum region.
\end{abstract}

\section{Introduction}

With the popularity of mushrooms as functional foods, its demand and consumption also increase. However, the perishable nature of mushrooms causes difficulty in their distribution and marketing as fresh produce (Li et al., 2013). Prevention of browning or discoloration after harvest has been studied for more than a decade now but still finding the most appropriate method of control is still not established. This may be because of the sensitivity of mushroom to its environmental factors of which it is grown, the morphological and cultural characteristics of various strains and the substrate from which it is cultivated. Also, once oyster mushroom deteriorated, its fruiting body can cause severe gastrointestinal discomfort when consumed (Hassan and Medany, 2012). In a study conducted by Lukasse and Polderdijk (2003), the postharvest life of mushroom is predominantly affected by temperature such that its postharvest life reduces from 9 days at $2^{\circ} \mathrm{C}$ to 3 days at $18^{\circ} \mathrm{C}$. Thus, extending postharvest storage is a constant quest for the production and supply chain of mushrooms.

Considering the highly perishable nature of oyster mushroom, the need to find an appropriate and most suitable processing technique that could prolong the shelf life without compromising the nutritional and phytochemical properties of the commodity is a challenge. Among all the available processing methods, vacuum frying seems to be the most viable solution to this problem. 
Vacuum frying is a technique of deep fat frying foods under pressure below atmospheric levels, which serves to reduce oil content, discoloration and losses of vitamins and other compounds normally associated with oxidation and high temperature processing (Diamante et al., 2015). It is an alternative method to produce a high quality fried product at lower processing temperature. Processing at low temperature and pressure reduces the amount of oil penetrating into the material and the deterioration and oxidation of the frying oil, resulting in stable fried food products (Sothornvit, 2011). Compared with other dehydration technologies for fruits and vegetables, vacuum frying is a viable option to obtain high quality dried products in much shorter processing time (Tarzi et al., 2011).

With the shift in the lifestyle of consumers, foods having a high amount of macro and micronutrient as well as high antioxidant properties are becoming popular this time. Oyster mushroom, which is one of those commodities containing both high amounts of nutrients and antioxidant properties is now given attention and thus preservation of its quality and improving its shelf life has been the subject of interest of health-conscious individuals. Thus, this study aimed to optimize the processing parameters for the production of a vacuum fried product from oyster mushroom (Pleurotus ostreatus (Jacquin) P. kummer).

\section{Materials and methods}

\subsection{Procurement and preparation of oyster mushroom}

Fruiting bags of oyster mushroom were procured from Mushroom Training Center, Capiz State University, Burias Campus, Mambusao, Capiz. It was opened and allowed to grow up to its desired fruiting body maturity. The freshly harvested oyster mushroom of 2, 3, and 4 days fruiting body maturity were trimmed to remove the unwanted parts then cleaned by wiping the mushroom with dry tissue paper to remove adhering dirt. Only samples of similar color, size and thickness were considered.

\subsection{Experimental treatment and design}

In order to determine the optimum processing conditions and formulation, a $3^{3}$ factorial experiment was employed following the Central Composite Design (CCD). The three variables considered were fruiting body maturity of oyster mushroom, salt concentration and frying time. A total of fifteen experimental combinations (Table 1) were considered for physicochemical, biochemical analyses and sensory evaluation.
Table 1. Experimental combinations for the process optimization of vacuum fried oyster mushroom

\begin{tabular}{|c|c|c|c|}
\hline 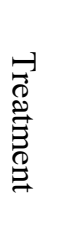 & 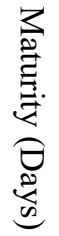 & 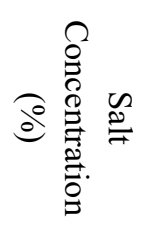 & 密 \\
\hline 1 & 2 & 2 & 25 \\
\hline 2 & 4 & 2 & 25 \\
\hline 3 & 4 & 4 & 25 \\
\hline 4 & 2 & 4 & 25 \\
\hline 5 & 2 & 2 & 35 \\
\hline 6 & 4 & 2 & 35 \\
\hline 7 & 4 & 4 & 35 \\
\hline 8 & 2 & 4 & 35 \\
\hline 9 & 3 & 2 & 30 \\
\hline 10 & 4 & 3 & 30 \\
\hline 11 & 3 & 4 & 30 \\
\hline 12 & 2 & 3 & 30 \\
\hline 13 & 3 & 3 & 35 \\
\hline 14 & 3 & 3 & 25 \\
\hline 15 & 2 & 2 & 25 \\
\hline
\end{tabular}

\subsection{Vacuum frying process of oyster mushroom}

Oyster mushrooms for vacuum frying were cleaned and trimmed for unwanted parts. It was blanched according to specified temperatures and blanching time. The blanched oyster mushrooms were soaked in brine for 3 mins and drained for 20 mins. Then it was placed and arranged in polyethene bags. Once the blanched oyster mushroom approximately attained uniform temperature, it was immediately placed inside the freezer for not less than twenty-four hours. After which, the frozen oyster mushroom was fried under vacuum at the specified frying time and frying temperature cooled and spun to remove excess oil from the product.

\subsection{Proximate analysis of oyster mushroom at different stages of maturity}

The proximate analysis (moisture, ash, protein, fat and fiber contents) of freshly harvested oyster mushroom at different stages of fruiting body maturity was done following the standard methods described in AOAC (2000). Carbohydrate content was calculated by subtracting the amount of moisture, ash, protein, fat and fiber content from 100 .

\subsection{Determination of total phenolics content}

The total phenolic content determination of fresh oyster mushroom was done following the procedure of Turnos (1993) as cited by (Tadeo et al., 2018) with slight modification. In brief, the sample was first subjected to 
ethanolic extraction. Approximately 5-7 g of fresh oyster mushroom was added with $30 \mathrm{~mL}$ of $80 \%$ ethanol. It was homogenized at high speed for 2 mins followed by boiling in water bath for 5 mins. The boiled mixture was centrifuged for 10 mins. After which the supernatant was collected and the residue was subjected to second extraction by adding $15 \mathrm{~mL}$ of $80 \%$ ethanol, boiled in water bath and centrifuged. The supernatant was again collected and mixed together with the first extraction and stored ready for analysis. Collected samples were analyzed spectrophotometrically using Folin Ciocalteau reagent. The oyster mushroom extract was diluted by adding $0.9 \mathrm{~mL}$ distilled water to $0.1 \mathrm{~mL}$ of extract. Then, $2 \mathrm{~mL}$ of $0.25 \mathrm{~N}$ Folin-Ciocalteau reagent was added. The solution was mixed and allowed to stand for 5 mins at room temperature. $\mathrm{Na}_{2} \mathrm{CO}_{3}$ solution $(1 \mathrm{~N})$ of 2 $\mathrm{mL}$ was added and mixed. The solution was allowed to stand for $1 \mathrm{hr}$ in the dark at room temperature. The absorbance of the final solution was read and recorded at $640 \mathrm{~nm}$ using a UV-Vis spectrophotometer. A standard curve was generated using concentrations of gallic acid stock solutions. Linear fit from regression analysis of the linear curve was used to compute the concentration of extracts coming from the sample. The total phenolic content was expressed as mg of gallic acid equivalent per $\mathrm{g}$ of oyster mushroom fresh weight (mg GAE. $\left.{ }^{-1}\right)$ and was calculated following the formula below:

$$
\mathrm{T}=\frac{\mathrm{C} \cdot \mathrm{V}}{\mathrm{M}}
$$

Where $\mathrm{T}=$ Total phenolic content, mg per $\mathrm{g}$ oyster mushroom, in GAE, $\mathrm{C}=$ Concentration of gallic acid established from the standard curve, $\mathrm{mg}$ per $\mathrm{mL}, \mathrm{V}=$ Volume of extract, $\mathrm{mL}$, and $\mathrm{M}=$ Weight of oyster mushroom sample, $g$

\subsection{Determination of radical scavenging activity}

The DPPH assay was based on the determination of scavenging free 1,1-diphenyl-2pidrylhydrazyl (DPPH) radicals. The method of Castro et al. (2012) as cited by Limbaga (2017) was followed. A DPPH solution was prepared by mixing $2.5 \mathrm{mg}$ of DPPH in $50 \mathrm{~mL}$ ethanol. A blank was prepared by mixing $1 \mathrm{~mL}$ of extraction solvent ( $80 \%$ ethanol) with $1 \mathrm{~mL}$ of DPPH solution. For sample analysis, $1 \mathrm{~mL}$ of the sample extract was mixed with $1 \mathrm{~mL}$ DPPH solution. The reaction was allowed to proceed for 30 mins at room temperature in the dark. The absorbance of the blank (Ao) and the sample extract (As) was measured at $517 \mathrm{~nm}$. The result was expressed in terms of percentage of DPPH reduction. Q refers to radical scavenging activity or inhibition and is defined by the following equation:

$$
Q=\frac{A o-A s}{A o} \times 100
$$

\subsection{Sensory evaluation}

Vacuum fried oyster mushrooms were subjected to sensory evaluation using the 9-point hedonic scale and quality scoring method. The product was evaluated based on their sensory attributes such as color, aroma, crispiness, taste, oiliness, aftertaste and general acceptability. The sensory evaluation was carried out following the set plan of incomplete block design with $\mathrm{t}=15, \mathrm{k}=7, \mathrm{~b}=15, \mathrm{I}=3, \mathrm{E}=0.92$, Type $\mathrm{I}$ (Cochran and Cox, 1957). There were four replications of the set plan to come up with 60 panelists, wherein each panel will only evaluate 7 out of 15 treatments making a total of twentyeight observations per treatment.

\subsection{Determination of the optimum experimental combinations}

Data gathered from the sensory evaluation, physicochemical tests and biochemical tests for all experimental treatments were analyzed employing the response surface regression with the use of Statistical Analytical Software (SAS). Parameters which were found not significant in the lack of fit test were considered for the identification of the optimum region. Using the Statistica version 6, a graphical presentation of the response surface plots was generated for all analyses of each run condition. Contour plots were superimposed to identify the optimum region of the experiment. The location of the optimum region was done by superimposing the contour plots of the radical scavenging activity (\% inhibition) ash, crude protein and crude fiber with cut-off values of $60 \%, 4 \%, 11 \%$ and $10 \%$ respectively.

\subsection{Statistical analysis}

Sensory and physico-chemical evaluation data were analyzed using SAS v6.12 and Statistica v.6 to determine the effects of independent variables and to generate surface and contour plots.

\section{Results and discussion}

\subsection{Proximate composition}

In a vacuum frying process, the food is heated under reduced pressure in a closed system that can lower the boiling points of both the oil and water in the fried food. In this case, the unbound water in fried food can be rapidly removed when the oil temperature reached the boiling point of water (Tarzi et al., 2011). The moisture content of vacuum fried oyster mushroom ranged from 3.51 to $8.17 \%$ with treatment 3 as the lowest and treatment 10 the highest with an overall response mean of $5.77 \%$. At $85^{\circ} \mathrm{C}$ frying temperature, the moisture contents obtained from different treatment combinations of vacuum fried oyster mushroom was similar with the 
moisture content of vacuum fried banana fried at $100^{\circ} \mathrm{C}$ for 25 mins (Ruttanadech and Chungcharoen 2015).

The response surface plots for moisture content (Figure 1 Aa) shows that at constant frying time, moisture content of vacuum fried oyster mushroom increases across the lowest and middle levels of salt concentrations. When the level of salt concentration was held constant (Figure 1Ab), the moisture content is at its highest at 4 days old maturity across any level of frying time. This suggests that maturity of oyster mushroom dictates the moisture content of the vacuum fried product. Similarly, when the maturity of oyster mushroom was held constant (Figure 1Ac), the moisture content increased when the frying time was at its lowest (25 mins) and the salt concentration was at 2 to $3 \%$. Technically, salt draws out moisture from the food by the process of osmosis, resulting in its low moisture content. This result is in accordance with the findings of Ruttanadech and Chungcharoen (2015) on the effects of temperature and time on the physical properties of banana by vacuum frying technique, they observed that the higher frying temperature and longer frying time provided the higher water evaporation which in turn resulted to lower moisture content.

The ash values obtained from different treatment combinations ranged from 3.13 to $9.39 \%$ with treatment 8 the lowest and treatment 7 the highest. Response surface regression analysis (Table 2) indicated that salt concentration and frying time have both linear and quadratic effect on the ash content of the vacuum fried oyster mushroom. Response surface plots as shown in Figure 2 shows that at constant frying time (Figure 1Ba), the ash content of vacuum fried oyster mushroom is highest when the concentration of salt is at maximum and the stage of maturity is also at a maximum. When the frying time was held constant, the highest ash content was observed when the maturity was again maximum across middle and high levels of frying time. Similarly, when the maturity was held constant (Figure 1Bc), the highest ash content occurred at maximum salt concentration across middle and maximum levels of frying time.

The results of the response surface regression analysis as presented in Table 2 shows that maturity significantly affects the crude protein content of vacuum fried oyster mushroom quadratically at 5\% level of significance. Also, the interaction between maturity and salt concentration significantly affected the protein content at $1 \%$ level of significance. The response surface plot for crude protein content of vacuum fried oyster mushroom shows that at constant frying time (Figure $1 \mathrm{Ca})$, the highest protein content is at 4 days old oyster mushroom and lowest level of salt concentration. This observation is consistent with characteristic of proteins which is easily denatured not just by heat but also by acid or alkali (Nielsen, 2003). As the salt concentration was held constant (Figure $1 \mathrm{Cb}$ ), highest crude protein content was observed at lowest level of maturity across any levels of frying time. Since the frying temperature employed during the vacuum frying process was only

Table 2. Summary of effect estimates of the proximate composition of vacuum fried oyster mushroom

\begin{tabular}{|c|c|c|c|}
\hline \multirow{2}{*}{ Variables } & \multicolumn{3}{|c|}{ Effect Estimates } \\
\hline & MCns & Ash & Crude Protein \\
\hline Maturity & $1.496600 \mathrm{~ns}$ & $-2.748488 n s$ & $-2.909852 \mathrm{~ns}$ \\
\hline Salt conc. & $1.391279 \mathrm{~ns}$ & $-7.733783 * *$ & $-0.630734 n s$ \\
\hline Frying time & $-0.099363 n s$ & $1.824189 * *$ & $1.448261 \mathrm{~ns}$ \\
\hline Maturity*Maturity & $0.011747 \mathrm{~ns}$ & $-0.006016 n s$ & $1.017031^{*}$ \\
\hline Salt conc. * Maturity & $-0.097083 n s$ & $0.601250 * *$ & $-0.756667 * *$ \\
\hline Salt conc. ${ }^{*}$ Salt conc. & $-0.565747 \mathrm{~ns}$ & $1.598641 * *$ & $0.434805 \mathrm{~ns}$ \\
\hline Frying time*Maturity & $-0.005417 \mathrm{~ns}$ & $0.094250 * *$ & $0.004333 \mathrm{~ns}$ \\
\hline Frying time* Salt conc. & $0.064487 \mathrm{~ns}$ & $-0.090225^{* *}$ & $0.013556 \mathrm{~ns}$ \\
\hline Frying time* Frying time & $-0.001978 n s$ & $-0.030930 * *$ & $-0.023040 \mathrm{~ns}$ \\
\hline \multirow{2}{*}{ Variables } & \multicolumn{3}{|c|}{ Effect Estimates } \\
\hline & Crude Fat & Crude Fiber & Carbohydrates \\
\hline Maturity & $38.613686^{*}$ & $-4.933255^{*}$ & $-29.518691 \mathrm{~ns}$ \\
\hline Salt conc. & $-48.257299 *$ & $-9.185895 * *$ & $64.416431 * *$ \\
\hline Frying time & $-4.942505 n s$ & $-1.335392 *$ & $3.104810 \mathrm{~ns}$ \\
\hline Maturity*Maturity & $-6.071670 *$ & $0.275029 \mathrm{~ns}$ & $4.773879 \mathrm{~ns}$ \\
\hline Salt conc. $*$ Maturity & $-1.696667 n s$ & $0.013750 \mathrm{~ns}$ & $1.935417 \mathrm{~ns}$ \\
\hline Salt conc.* Salt conc. & $7.290872 *$ & $1.402614 * *$ & $-10.161185 * *$ \\
\hline Frying time*Maturity & $0.047667 \mathrm{~ns}$ & $0.120750 * *$ & $-0.261583 \mathrm{~ns}$ \\
\hline Frying time* Salt conc. & $0.303530 \mathrm{~ns}$ & $0.039893 \mathrm{~ns}$ & $-0.331241 \mathrm{~ns}$ \\
\hline Frying time*Frying time & $0.064064 \mathrm{~ns}$ & $0.013089 \mathrm{~ns}$ & $-0.021205 n s$ \\
\hline
\end{tabular}

*significant $(\mathrm{p}<0.05) \quad * *$ significant $(\mathrm{p}<0.01) \quad$ ns-not significant 
A.
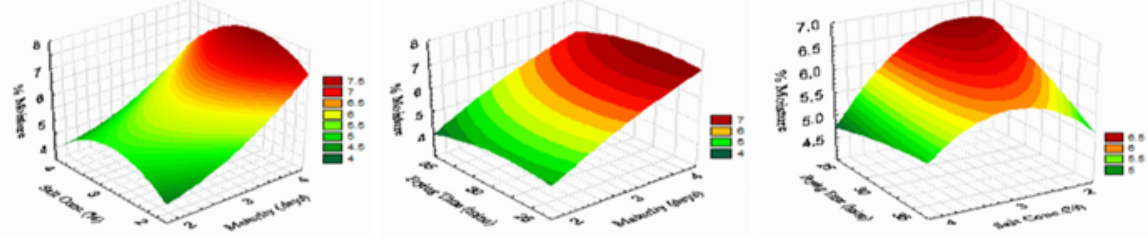

B.
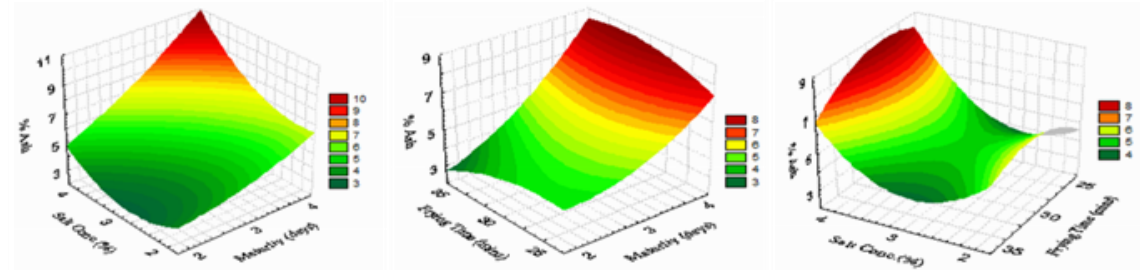

C.
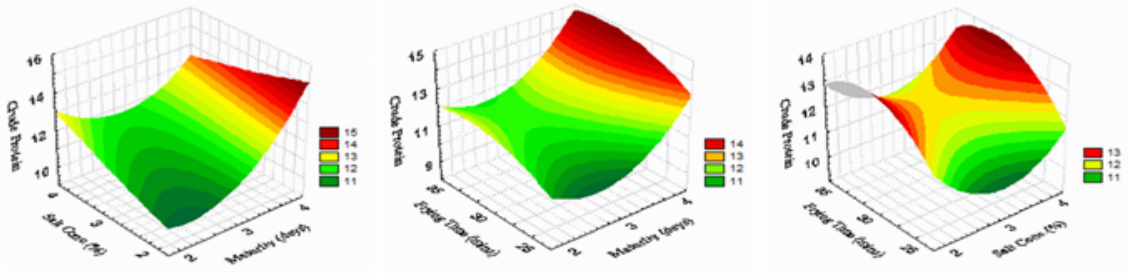

D.
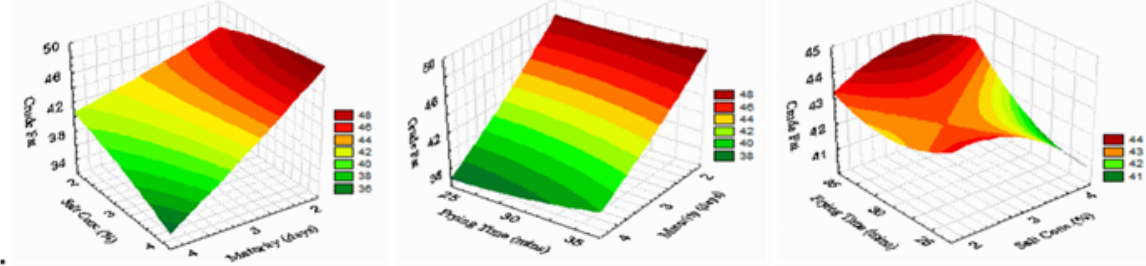

E.
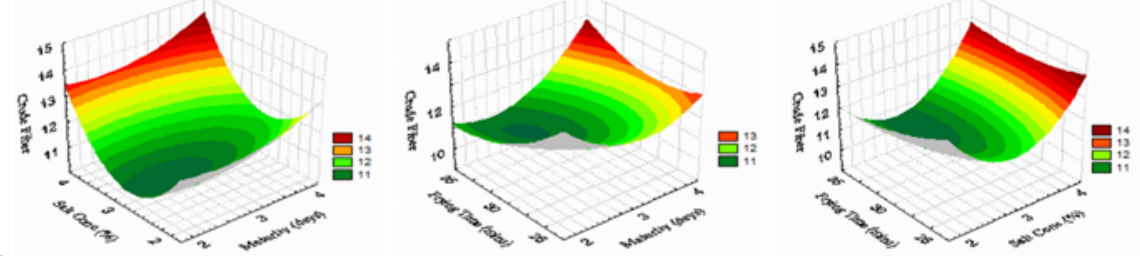

F.

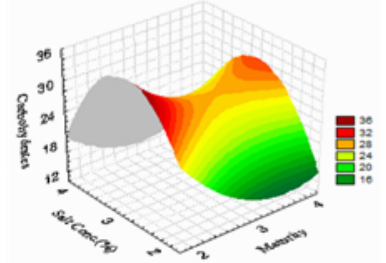

(a)

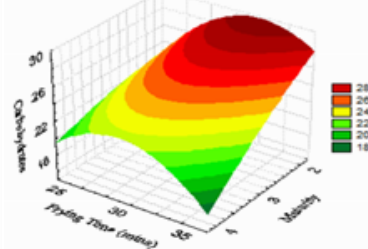

(b)

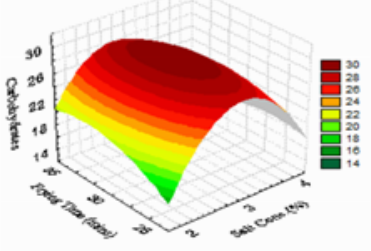

(c)

Figure 1. Surface plots for A- moisture, B- ash, C- crude protein, D- crude fat, E- crude fiber and F- carbohydrates content of vacuum fried oyster mushroom at constant (a) frying time, (b) salt concentration and (c) maturity

$85^{\circ} \mathrm{C}$, frying time as illustrated in the figure did not significantly affect the protein content of the finished product. Moreover, at constant maturity, the crude protein content increased at middle level of frying time across both high and low levels of salt concentration (Figure 1Cc).

The amount of fat varies from 35.62 to $49.35 \%$ with an overall response mean of $40.92 \%$. Treatment 3 (4 days maturity, $4 \%$ salt concentration and 25 mins frying time) while treatment 8 ( 2 days maturity, $4 \%$ salt concentration and 35 mins frying time). This result is supported by the findings of Maity et al. (2014) in their study on the effect of vacuum frying on changes in quality attributes of jackfruit bulb slices in which they found out that oil content of vacuum fried jackfruit increased with an increase in the frying time. This implies that the longer is the frying time, the more oil will be absorbed by the fried food material.

The response surface plots for the crude fat content of vacuum fried oyster mushroom shows that at constant 
frying time (Figure 1Da), the crude fat content is at its highest when the maturity of oyster mushroom is at its lowest and salt concentration is at the middle and highest level. On the other hand, when the salt concentration was held constant (Figure 1Db), the highest crude fat content was still at 2 days old maturity across any levels of frying time. As the constant shifted to maturity, the graph (Figure 1Dc) shows that the highest crude fat content was still the 2 days old maturity across high and low levels of frying time. This finding implies that maturity and salt concentration really affect significantly the crude fat content of vacuum fried oyster mushroom.

Dietary fiber is the sum of the non-digestible components of the foodstuff or food product. The crude fiber content of vacuum fried oyster mushroom ranged from 9.86 to $13.72 \%$ with an overall response mean of $11.94 \%$. The highest crude fiber was from the vacuum fried oyster mushroom made from 4 days old mushroom, $4 \%$ salt concentration and 35 mins frying time while treatment 12 made from 2 days old oyster mushroom, $3 \%$ salt concentration and 30 mins frying time had the lowest. The response surface regression reveals that maturity and frying time had linear significant effect on the crude fiber content of the vacuum fried oyster mushroom. Salt concentration on the other hand, had both significant linear and quadratic effect on the crude fiber content as well as the interaction between frying time and maturity. Response surface plot shows that at constant frying time (Figure 1Ea), the highest crude fat content was at maximum stage of maturity and at highest level of salt concentration. Whereas, at constant salt concentration (Figure 1Eb), the crude fiber content was at its highest at maximum stage of maturity and highest level of frying time. At constant maturity (Figure 1Ec), the highest crude fiber was attained when salt concentration was at maximum level across any levels of frying time.

The amount of carbohydrates of vacuum fried oyster mushroom from different treatment combinations vary ranging from 17.1 to $53.38 \%$. The highest carbohydrates contents are those made from treatment 12 while the lowest are those from treatment 4 . Response surface regression analysis (Table 2) shows that salt concentration had highly significant linear and quadratic effect on the carbohydrates content of vacuum fried oyster mushroom. This means that salt concentration significantly influenced the carbohydrate content of the finished product. The interaction between variables shows that at constant frying time (Figure $1 \mathrm{Fa}$ ), the amount of carbohydrates increased at middle level of salt concentration at both high and low level of maturity. As the constant was shifted to salt concentration (Figure $1 \mathrm{Fb})$, carbohydrates content was at its highest at the lowest level of maturity across 39 mins of frying time. At constant maturity (Figure $1 \mathrm{Fc}$ ) however, the amount of carbohydrates increased at both middle levels of salt concentration and frying time.

\subsection{Total phenolics content}

Phytochemicals such as phenolics and flavonoids play an important role in increasing health benefits (Simoes et al., 2009). The mean total phenolics content (mg GAE/g sample) and radical scavenging activity (\% inhibition) of oyster mushroom as affected by different levels of maturity, salt concentration and frying time are presented in Table 3. After vacuum frying, Treatment 11 (3 days old maturity, 4\% salt concentration and 30 mins frying time) had the highest retention of total phenolics of $0.4766 \mathrm{mg} \mathrm{GAE} / \mathrm{g}$ sample while treatment 10 (4 days maturity, $3 \%$ salt concentration and 30 mins frying time) had the lowest retention amounting to only $0.2998 \mathrm{mg}$ GAE/g sample.

Table 3. Mean total phenolics content (mg/g GAE) and radical scavenging activity (\% inhibition) of oyster mushroom of as affected by different levels of maturity, salt concentration and frying time

\begin{tabular}{|c|c|c|c|c|c|}
\hline 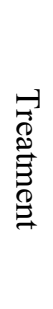 & 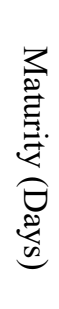 & 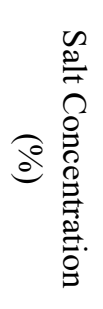 & 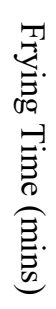 & 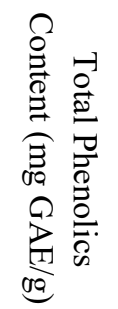 & 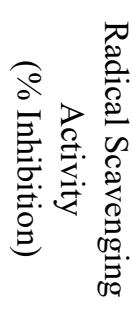 \\
\hline 1 & 2 & 2 & 25 & 0.4032 & 32.2 \\
\hline 2 & 4 & 2 & 25 & 0.4149 & 45.76 \\
\hline 3 & 4 & 4 & 25 & 0.344 & 60.07 \\
\hline 4 & 2 & 4 & 25 & 0.4505 & 66.19 \\
\hline 5 & 2 & 2 & 35 & 0.3799 & 57.07 \\
\hline 6 & 4 & 2 & 35 & 0.4692 & 46.88 \\
\hline 7 & 4 & 4 & 35 & 0.398 & 52.64 \\
\hline 8 & 2 & 4 & 35 & 0.333 & 91.01 \\
\hline 9 & 3 & 2 & 30 & 0.3715 & 85.61 \\
\hline 10 & 4 & 3 & 30 & 0.2998 & 75.3 \\
\hline 11 & 3 & 4 & 30 & 0.4766 & 86.45 \\
\hline 12 & 2 & 3 & 30 & 0.4701 & 82.01 \\
\hline 13 & 3 & 3 & 35 & 0.4571 & 84.65 \\
\hline 14 & 3 & 3 & 25 & 0.4886 & 58.27 \\
\hline 15 & 3 & 3 & 30 & 0.4812 & 87.65 \\
\hline
\end{tabular}

Response surface regression analysis revealed that maturity had both significant linear and quadratic effect on the total phenolics content of the vacuum fried product. Initially, the total phenolics content of oyster mushroom at their corresponding maturity was higher than values obtained from the vacuum dried product. The decrease may be due to the leaching out of water-soluble phenols during the frying process (Kita et al., 2013). The 
minimum loss in total phenolics could be attributed to the application of vacuum during frying which could retain maximum amounts of phytochemicals in the fried material (Maity et al., 2014). Therefore, the phenolics content of oyster mushroom is inversely related to maturity. As the age of mushroom increased, the phenolic content decreased. Likewise, the interaction between frying time and quadratic effect on the phenolic content of the vacuum fried product from oyster mushroom.

The response surface plots of the total phenolic content of vacuum fried oyster mushroom show that at constant frying time (Figure 2Aa), the highest phenolic content was attained at the middle stage of maturity across all levels of salt concentration. Similarly, at constant salt concentration, the total phenolic content was at its highest at the middle stage of maturity across any levels of frying time (Figure 2Ab). This illustrates the result of the regression analysis on the significant effect of maturity on the total phenolic content of the vacuum fried product. Figure $2 \mathrm{Ac}$ shows that middle levels of both frying time and salt concentration attained the highest total phenolic content.

\subsection{Antioxidant activity}

The radical scavenging activity (RSA) of the vacuum fried oyster mushroom was determined by DPPH assay and results are presented in Table 3 . The values obtained for the antioxidant presented as \% inhibition ranged from $32.19 \%$ to $91.01 \%$ with an overall response mean of $66.76 \%$.

The response surface regression analysis as shown in Table 4, showed that maturity caused a quadratic significant effect on the antioxidant activity of vacuum fried oyster mushroom while other variables are not significant. This means that only maturity affected the antioxidant activity of the finished product.

Figure 2B presents the response surface plots of the antioxidant activity of vacuum fried oyster mushroom. Figure $2 \mathrm{Ba}$ shows that at constant frying time, the antioxidant activity increased at the middle stage of maturity and maximum level of salt concentration. At constant salt concentration (Figure 2Bb), antioxidant activity was at its highest when the middle stage of maturity across the maximum level of frying time. On the other hand, at constant maturity (Figure 2Bc), the antioxidant activity was at its highest at the middle level of salt concentration and maximum level of frying time.

Table 4. Summary of effect estimates of the biochemical properties of vacuum fried oyster mushroom

\begin{tabular}{lcc}
\hline Variables & $\begin{array}{c}\text { Total Phenolics } \\
\text { Content }\end{array}$ & $\begin{array}{c}\text { Radical Scavenging } \\
\text { Activity }\end{array}$ \\
\hline Intercept & $-0.833164 \mathrm{~ns}$ & $-553.933593^{*}$ \\
Maturity & $0.341507^{*}$ & $116.131616 \mathrm{~ns}$ \\
Salt conc. & $-0.02449 \mathrm{~ns}$ & $101.667787 \mathrm{~ns}$ \\
Frying time & $0.069343 \mathrm{~ns}$ & $18.021293 \mathrm{~ns}$ \\
Maturity*Maturity & $-0.081853^{* *}$ & $-10.158251^{*}$ \\
Salt conc. ${ }^{*}$ Maturity & $-0.018046 \mathrm{~ns}$ & $-5.983208 \mathrm{~ns}$ \\
Salt conc.* Salt conc. & $0.024040 \mathrm{~ns}$ & $-8.630873 \mathrm{~ns}$ \\
Frying time*Maturity & $0.006405^{* *}$ & $-1.400475 \mathrm{~ns}$ \\
Frying time* Salt conc. & $-0.001127 \mathrm{~ns}$ & $-0.729343 \mathrm{~ns}$ \\
Frying time*Frying time & $-0.001458^{*}$ & $-0.160318 \mathrm{~ns}$ \\
\hline *significant (p<0.05), & $* *$ significant & $(\mathrm{p}<0.01), \quad \mathrm{ns}$-not \\
significant & &
\end{tabular}

\subsection{Sensory qualities of vacuum-fried oyster mushroom}

In terms of color of vacuum fried oyster mushroom, treatments described as light brown to brown were those that come from 4 days old fruiting bodies and fried for 35 mins. The brown color was attributed to the length of frying time and maturity of the oyster mushroom. As the oyster mushrooms matured, its color also changed from (a)

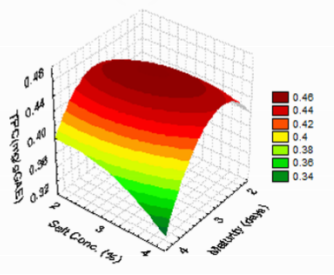

A.
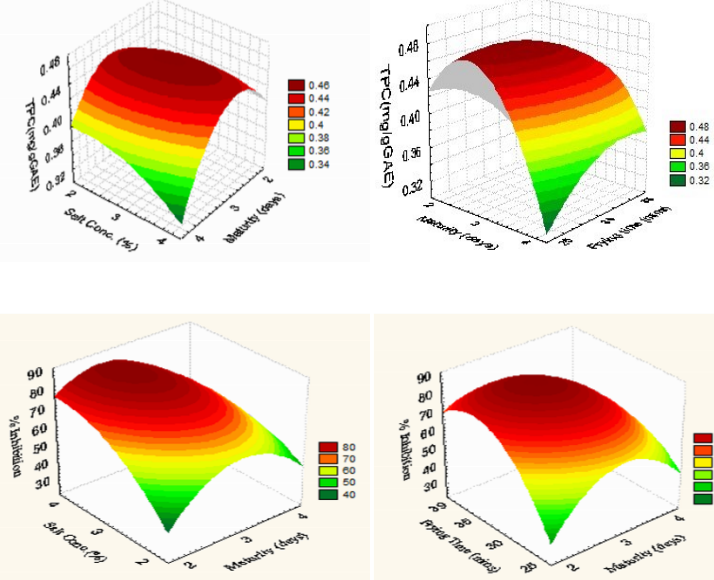

(b)

(c)
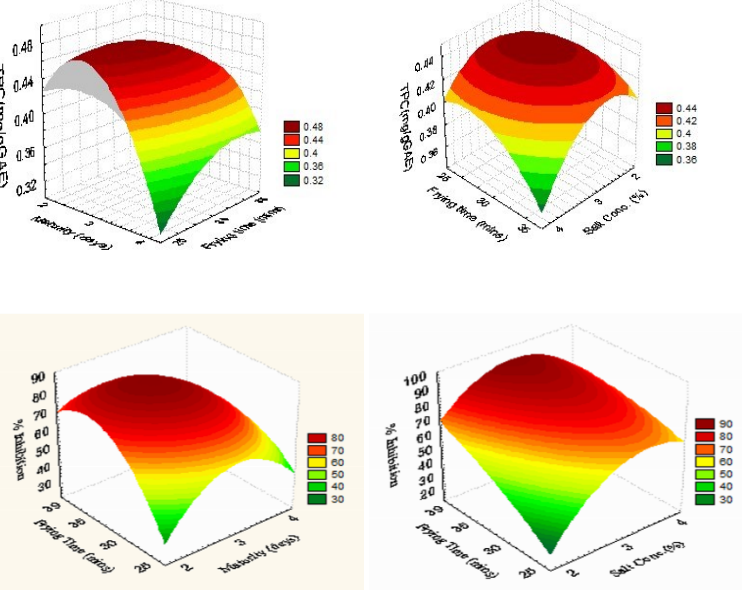

Figure 2. Surface plots for (A) total phenolics content (mg GAE/g sample) and (B) Radical Scavenging Activity (\% Inhibition) of vacuum fried oyster mushroom at constant (a) frying time, (b) salt concentration and (c) maturity 
white to brownish thus obtaining a darker color. The browning of mushroom occurs when they are subjected to forces that can disrupt cellular integrity such as vibrations, rough handling and ageing (Gao et al., 2014). According to Andres Bello et al. (2011) as cited by Diamante et al. (2015) the frying time-temperature combination of the cooking process are the main factors that influence the quality of fried products.

The color acceptability of the different treatments, ranges from 6.79 to 7.39 , with an overall response mean of 7.12 and falls within the range of "like moderately" to like very much" categories in the 9-point Hedonic scale. The highest ratings are obtained from those treatments which were fried for 35 mins or those which were harvested after four days fruiting body maturity. Treatment 2 with a combination of 4 days old fruiting body maturity, $2 \%$ salt concentration and 25 mins frying time together with Treatment 13 having 3 days old maturity, 3\% salt concentration and 35 mins frying time both obtained the highest acceptability rating of 7.39 while Treatment 1 , with 2 days old maturity, $2 \%$ salt concentration and 25 mins frying time obtained the lowest with an acceptability rating of 6.79 . The predicted value at stationary point is 7.18 and is slightly higher than the observed overall response mean of 7.12 and is considered as a saddle point. However, effect estimates for color revealed no significant effect on the color acceptability of the vacuum-fried product. The surface plots of the acceptability ratings for color of vacuum fried oyster mushroom shows that at constant frying time (Figure 3Aa), the color acceptability increases as the levels of salt concentration and maturity increases. This is because consumers usually associate the brown color to good quality fried product. Moreover, at constant salt concentration (Figure $3 \mathrm{Ab}$ ), color acceptability increases as the frying time is prolonged and the maturity is increased. However, at constant maturity (Figure 3Ac), the color acceptability increases when the frying temperature increases across middle level of salt concentration.

Aroma of a food reaches the nose before a food reaches the mouth, and so it plays an important role in determining its acceptability. Highly perceptible aroma was produced from Treatment 2 with 2 days old fruiting body maturity, $2 \%$ salt concentration and fried for 25 mins. Other treatments had aroma described as moderately perceptible except for treatments 6 and 14 which were described as slightly perceptible mushroom aroma. Treatment 10, which used 4 days old fruiting body maturity, 3\% salt concentration and 30 mins frying time, obtained the highest acceptability rating of 7.21 falls within the "like moderately" to "like very much category." On the other hand, vacuum fried oyster mushroom with 2 days fruiting body maturity, $2 \%$ salt concentration and 25 mins frying time had highly to moderately perceptible aroma and obtained the lowest acceptability ratings of 6.36 and fall within the like slightly to like moderately category of the 9-point Hedonic scale. The surface plots for the aroma acceptability shows that at constant frying time (Figure $3 \mathrm{Ba}$ ), the aroma acceptability was at its highest when the salt concentration was at mid-level across high and low levels of maturity. At constant salt concentration (Figure $3 \mathrm{Bb}$ ), the aroma acceptability increased with an increasing frying time and maturity while at constant maturity level (Figure 3Bc), the acceptability was at its highest when the frying time was at its highest at both low and middle level of salt concentration. This implies that the level of salt concentration influenced the aroma acceptability up to $3 \%$. Beyond $3 \%$ salt concentration, the aroma acceptability declined. The effect estimate for aroma revealed that salt concentration significantly affected the aroma acceptability of the vacuum fried oyster mushroom at 5\% level of significance. The increase in the frying time resulted in an increase in its acceptability but its increase is not enough to cause a significant difference on the resulting aroma acceptability.

One important quality parameter of desirable textural characteristics is crispiness because it implies freshness and high quality (Omidiran et al., 2015). The vacuumfried oyster mushrooms description ranged from not crispy to moderately crispy. The vacuum fried oyster mushroom produced from 4 days old fruiting body, $4 \%$ salt concentration and fried for 25 mins was described as not crispy by the panelists. The rest of the treatments were either described as slightly crispy to moderately crispy. Acceptability ratings of the different treatments show that Treatment 13, made from 3 days old oyster mushroom, 3\% salt concentration and fried for $35 \mathrm{~min}$ obtained the highest rating of 7.39 and fall within the like moderately to like very much category of the 9-point hedonic scale. On the other hand, the vacuum fried oyster mushroom which was described as not crispy obtained the lowest rating of 5.86 and fall within the neither like nor dislike to like slightly category. The effect estimates for crispiness showed that crispiness acceptability was affected by the salt concentration both linearly and quadratically. As the salt concentration increases, crispiness acceptability decreases. The salt tends to draw out the moisture from the oyster mushroom thereby producing a crispy but slightly spongy vacuum fried product. This implies that a oneunit increase in the level of salt concentration resulted in a corresponding decrease in the crispiness acceptability by 0.37698 units. The spongy texture made the product difficult to chew and shear thus resulting in a decrease in 
its acceptability. The response surface plots for crispiness acceptability shows that at constant frying time (Figure $3 \mathrm{Ca}$ ), the acceptability was at its highest when the level of oyster mushroom maturity was at its lowest across the middle level of salt concentration. This means that the immature oyster mushroom produced the most acceptable crispiness among sensory panelists. Figure $3 \mathrm{Cb}$ which showed the crispiness acceptability at constant salt concentration also revealed that 2 days old oyster mushroom had the highest acceptability across middle to the highest level of frying time. At constant maturity (Figure $3 \mathrm{Cc}$ ) however, the highest acceptability was favored when the concentration of salt was $3 \%$ and the frying time was at its highest.

Taste description responses from panelists differ from just right to slightly salty. Generally, the limiting factor for the taste acceptability of vacuum-fried oyster mushroom was the level of salt, with those having the highest percentage of salt described as slightly salty. The acceptability rating ranged from 6.61 to 7.36 with an overall response mean of 7.16 almost similar with the predicted value at station point which was 6.17 and a saddle point. The value obtained fall within the like slightly to like very much category of the 9-point hedonic scale. Response surface regression analysis revealed no significant difference among treatment means. However, the interaction effect between frying time and maturity caused a significant effect on the taste acceptability of vacuum-fried oyster mushroom at 5\% level of significance. The interaction between variables shows that taste acceptability is high when the maturity is 2 days old across any levels of salt concentration (Figure 3Da) while at constant salt concentration (Figure $3 \mathrm{Db}$ ), acceptability was increased when the frying time was at its highest and the level of maturity is at its lowest. When the constant was shifted to maturity, the taste acceptability was at its highest at $3 \%$ salt concentration across $35 \mathrm{~min}$ of frying time (Figure 3Dc).

In terms of oiliness, all the treatments were described as slightly oily except for treatment 4 which was made from 2 days old oyster mushroom with 4\% salt concentration and fried for 25 mins which was described as not oily. According to Maitey et al. (2014), the increase in frying temperature and time during vacuum frying also resulted to an increase in the oil content. Thus, the lowest frying time produced a product which was described as not oily. Acceptability ratings on the other hand, shows that the highest oiliness acceptability rating was obtained from treatment 13 made from 3 days old oyster mushroom with $3 \%$ salt concentration and fried for 35 mins with a rating of 7.61 and falls within the like moderately to like very much category. The lowest acceptability rating on the other hand was obtained from treatments 2 and 3 which were both produced from 4 days old oyster mushroom and fried for 25 mins with a rating of 7.0 still within the like moderately category. Response surface regression analysis revealed no significant linear and quadratic effects. This means that different frying times did not significantly affect the oiliness acceptability of the vacuum-fried oyster mushroom. The response surface plots show that at constant frying time (Figure 3Ea), the highest acceptability rating was observed at the highest level of salt concentration across the lowest level of maturity. At constant salt concentration (Figure 3Eb), the acceptability was at its highest when the frying time was at its highest and the salt concentration was at the middle level of $3 \%$. When the constant was shifted to the level of maturity (Figure 3Ec), the acceptability rating increased at the highest level of frying time across 3\% salt concentration.

The quality descriptions for aftertaste vary from slightly to moderately perceptible mushroom aftertaste. Generally, vacuum-fried oyster mushroom produced from 2 days old oyster mushroom had slightly perceptible aftertaste while those produced from 4 days old had moderately perceptible aftertaste. This suggests that maturity of oyster mushroom affected the aftertaste acceptability of the resulting product. As shown in the response surface plot for the aftertaste acceptability of vacuum-fried oyster mushroom at constant frying time (Figure $3 \mathrm{Fa}$ ), the acceptability was at its highest at the lowest level of maturity across any levels of salt concentration while at constant salt concentration (Figure $3 \mathrm{Fb}$ ), the acceptability rating increased at both high and low level of frying time across low to middle level of maturity. At constant maturity (Figure $3 \mathrm{Fc}$ ), acceptability increased at both high and low frying time at middle level of salt concentration.

When evaluating food product, the acceptability of all the sensory attributes or the general acceptability is used as the basis for acceptance. The observed general acceptability of the product ranges from 6.82 to 7.79 with an overall response mean of 7.14 falls within like moderately to like very much of the 9-point hedonic scale. Treatment 13 had the highest general acceptability rating among the treatments. This treatment also had the highest acceptability rating for color, crispiness, oiliness and aftertaste. This implies that sensory panelists prefer a level combination of treatment 15 among other treatments. Effect estimates, however, revealed that different variables have no significant effect on the general acceptability of the vacuum-fried product. Interaction and relationship of the different variables show that at constant frying time (Figure 3Ga), the acceptability increases at middle level of maturity (3 
A.

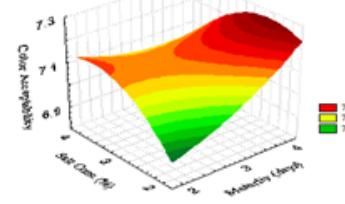

를
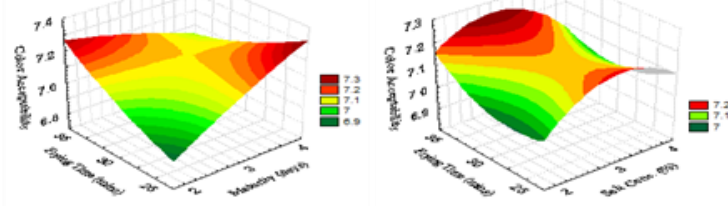

B.
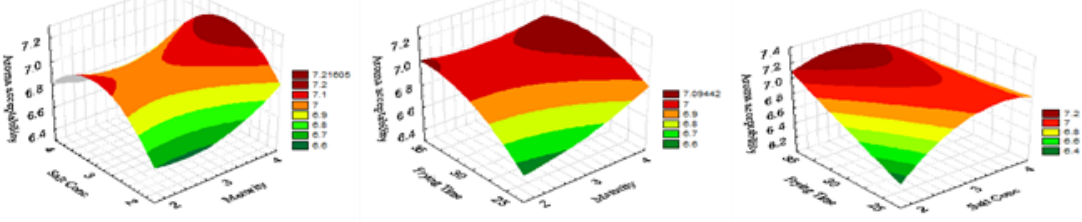

C.
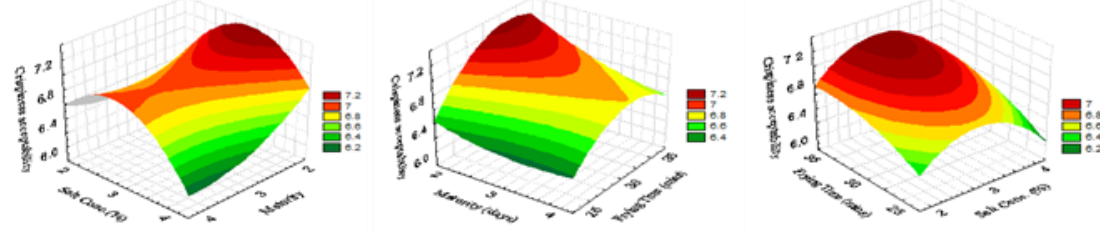

D.
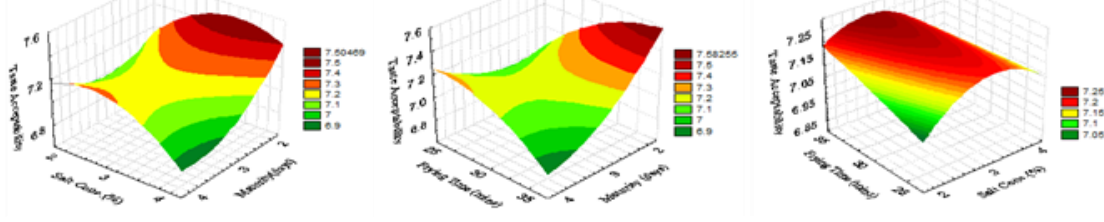

E.

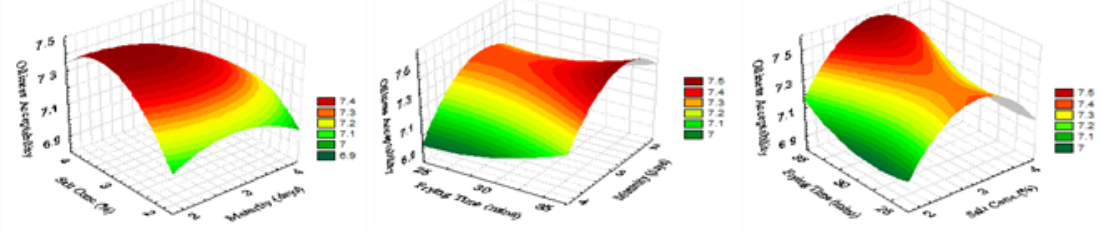

F,

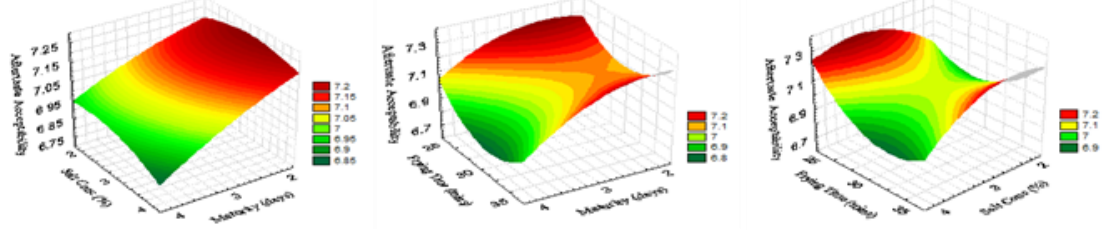

G.

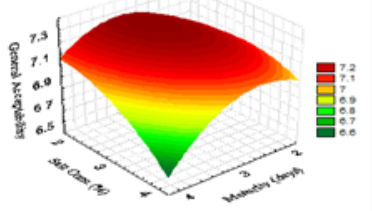

(a)

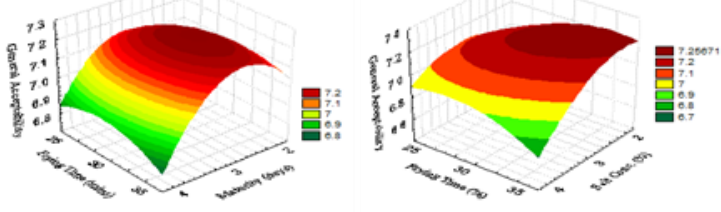

(c)

Figure 3. Surface plots for A- color, B- aroma, C- crispiness, D- taste, E- oiliness, F- aftertaste and G- general acceptability of vacuum fried oyster mushroom at constant (a) frying time, (b) salt concentration and (c) maturity

days) and at low to middle level of salt concentration (2$3 \%)$. General acceptability also increases at middle level of maturity across any levels of frying time when salt concentration was held constant (Figure 3Gb). When maturity was kept constant (Figure 3Gc), general acceptability increases low to middle level of salt concentration $(2-3 \%)$ and at medium to high levels of frying time (30-35 $\mathrm{min})$.

\subsection{The optimized region for the most favorable process variables}

Optimum region refers to the most acceptable and most favorable process or formulation. Another parameter considered was the acceptability scores of all the sensory attributes of the vacuum fried oyster mushroom being evaluated having the same factor held constant. The contour plots were obtained using the mean values of the attributes tested. It gave an idea as to which levels of frying time, salt concentration and maturity combinations would result to a better quality product. To further limit the optimum region, a cut off score of 6.75 was set as the minimum. The shaded region represents values corresponding to scores of $\geq 6.75$ which fall in the "like slightly" to "like moderately" category in the 9 point Hedonic scale. Any point within the region indicates optimized combinations of the 
processing variables. At constant frying time (Figure 4a) superimposed graph shows the acceptable region (shaded region) which correspond to $3 \%$ salt concentration and a frying time of 30 to 35 mins.

At constant frying time (Figure 4b), the superimposed plot shows that the optimum region consists of 3\% salt concentration and a maturity of 3 to 4 days old while at constant maturity (Figure 4c), the optimum region is between 30 to 35 mins frying time and $3 \%$ salt concentration. Having all these considerations, the optimum combination has been identified to be 3 days maturity, $3 \%$ salt concentration and 35 mins frying time.

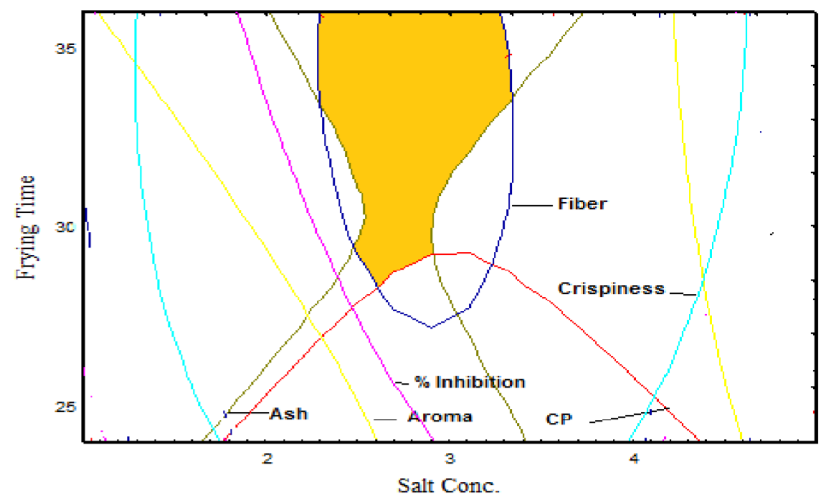

(a)

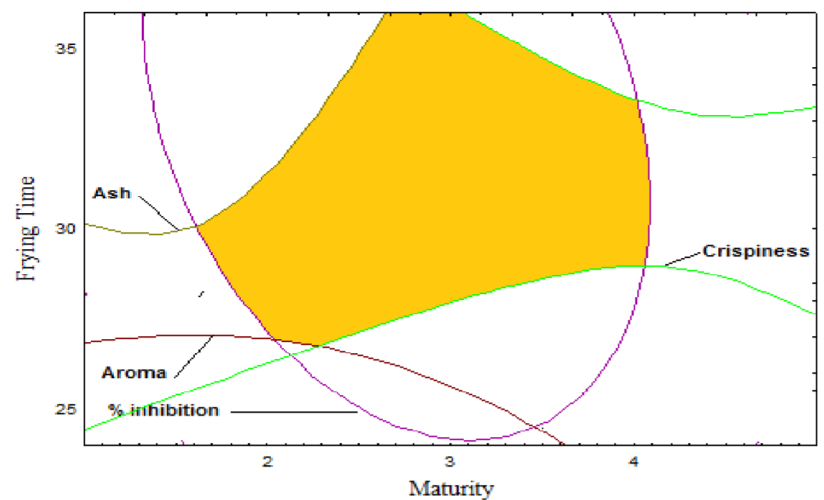

(b)

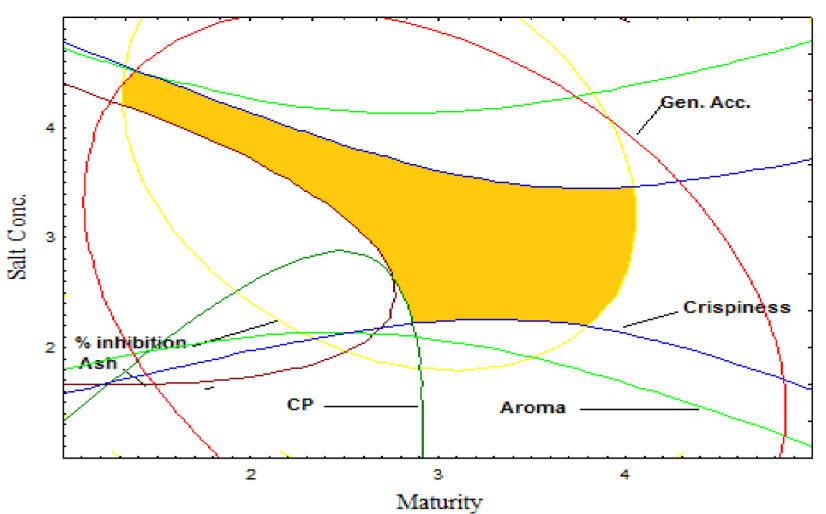

(c)

Figure 4. Acceptable regions (shaded) for vacuum fried oyster mushroom obtained by superimposing contour plots of scavenging activity (\% inhibition) ash, crude protein, crude fiber and sensory acceptability $>6.75$ with constant (a) 35 mins frying time, (b) 3\% salt concentration and (c) 3 days fruiting body maturity

\section{Conclusion}

Maturity of oyster mushroom significantly affected the crude fat, crude fiber and total phenolics content of the vacuum fried product. Salt concentration significantly affected the ash, crude fat, crude fiber, carbohydrates, aroma and crispiness. The product's ash and crude fiber contents were also significantly affected by the frying time. Superimposed contour plots showed that the optimum region based on a set of criteria and sensory acceptability is at the combination of 3 days fruiting body maturity, $3 \%$ salt concentration and $35 \mathrm{~min}$ frying time.

\section{Acknowledgement}

The authors would like to thank the Department of Science and Technology Accelerated Science and Technology Human Resource Development Program (DOST-ASTHRDP) for financial support.

\section{References}

Andres-Bello, A., Garcia-Segovia, P. and MartinezMonzo, J. (2011). Vacuum Frying: An Alternative to Obtain High-Quality Dried Products, 3(2), 63-78. http://doi.org/10.1007/s12393-011-9037-5" http:// doi.org/10.1007/s12393-011-9037-5

Cochran, W.G. and Cox, G.M. (1957) Experimental Design. 2nd ed., p. 615. New York, USA: John Wiley and Sons.

Diamante, 1.M., Shi, S., Hellmann, A. and Busch, J. (2015). Vacuum frying foods: Products, process and optimization. International Food Research Journal, 22(1), 15-22.

Hassan, F.R.H. and Mendaney, G.M. (2014). Effect of pre-treatments and drying temperatures on the quality of dried Pleurotus mushroom spp. Indian Journal of Horticulture, 69(4), 586-590.

Kita, A., Backowska-Barczak, A., Hamous, K., Kulakowska, K. and Lisinska, G. (2013). The effect of frying on anthocyanin stability and antioxidant activity of crisps from red and purple-fleshed potatoes (Solanum tuberosum L.), Journal of Food Composition and Analysis, 32(2), 169-175. https:// doi.org/10.1016/j.jfca.2013.09.006

Li, P., Zhang, X., Hu, H., Sun, Y., Wang, Y. and Zhao, Y. (2013). High carbon dioxide and low oxygen storage effects on reactive oxygen species metabolism in Pleurotus eryngii. Postharvest Biology and Technology, 85, 141-146. http:// doi.org/10.1016/j.postharvbio.2013.05.006

Limbaga, J.C. (2017). Physicochemical and Biochemical Changes of Yacon (Smallanthus sonchifolius) During Storage and Processing. Laguna, Philippines: 
UPLB, PhD Dissertation.

Lukasse, L.J.S. and Polderdijk, J.J. (2003). Predictive modelling of post-harvest quality evolution in perishables, applied to mushrooms. Journal of Food Engineering, 59(2-3), 191- $198 . \quad \mathrm{http}: / /$ doi.org/10.1016/S0260-8774(02)00458-2

Maity, T., Bawa, A.S. and Raju, P.S. (2014). Effect of Vacuum Frying on Changes in Quality Attributes of Jackfruit (Artocarpus heterophyllus) Bulb Slices. International Journal of Food Science, 2014, 752047. http://doi.org/10.1155/2014/752047

Nielsen, S.S. (2003). Food Analysis. Analyst, 21(1896), 255b-257. http://doi.org/10.1039/an896210255b

Omidiran, N.A., Sobukola, O., Sanni, A., Adebowale, A. -R.A., Obadina, O.A., Sanni, L.O., Tomlins, K. and Wolfgang, T. (2015). Optimization of some processing parameters and quality attributes of fried snacks from blends of wheat flour and brewer's spent cassava flour. Journal of Food Science and Nutrition, 4(1), 80-88. https://doi.org/10.1002/ fsn 3.255

Ruttanadech, N. and Chungcharoen, T. (2015). Effects of Temperature and Time on the Physical Properties of Banana by Vacuum Frying Technique presented at the International Conference on Advances in Agricultural, Biological and Environmental Sciences, July 22-23, 2015, p. 46-49. London, United Kingdom.

Simoes. A.D.N, Tudela, J.A., Allende, A., Puschmann, R. and Gil, M. (2009). Edible coatings containing chitosan and moderate modified atmosphere maintain quality and enhance phytochemicals of carrot sticks. Postharvest Biology and Technology, 51, 364-337. https://doi.org/10.1016/ j.postharvbio.2008.08.012

Sothornvit, R. (2011). Edible coating and post-frying centrifuge step effect on quality of vacuum-fried banana chips. Journal of Food Engineering, 107(34), 319-325. http://doi.org/10.1016/ j.jfoodeng.2011.07.010

Tadeo, P.R.M., Castillo-Israel, K.A.T., Serrano, E.P., Gandia, J.B.L. and Absulio, W.L. (2018). Physiological responses and storage quality of freshcut red and white dragon fruit (Hylocereus spp.) treated with 1-methylcyclopropene (1-MCP). International Food Research Journal, 25(5), 20902098

Tarzi, B.G., Bassiri, A. and Bamenimoghadam, M. (2011). Process Optimization in Vacuum Frying of Mushroom Using Response Surface Methodology. World Applied Sciences Journal, 14(7), 960-966. 